Conclusions: Low levels of MMP-7 and FGA at baseline were associated with better clinical outcome in eRA patients. Following further characterisation, such biomarkers would be of high clinical relevance for the optimisation of treatment of RA.

Disclosure of Interest: K. Hambardzumyan: None declared, C. Hamsten: None declared, H. Idborg: None declared, L. Lourido: None declared, S. Saevarsdottir: None declared, P. Nilsson: None declared, R. van Vollenhoven Grant/research support from: AbbVie, BMS, GSK, Pfizer, UCB, Consultant for: AbbVie, AstraZeneca, Biotest, BMS, Celgene, GSK, Janssen, Lilly, Novartis, Pfizer, UCB, P.-J. Jakobsson: None declared

DOI: 10.1136/annrheumdis-2018-eular.5408

\section{FRI0046 PATIENTS PRESENTING WITH NEW MUSCULOSKELETAL SYMPTOMS IN THE WRISTS, HANDS AND FEET ENRICHES DETECTION OF ANTI- CCP ANTIBODIES IN PRIMARY CARE - A NATIONAL COHORT STUDY}

P. Pentony $^{1,2}$, K. Karkalemis ${ }^{1}$, J.L. Nam ${ }^{1,2}$, K. Mankia ${ }^{2,3}$, L. Hunt ${ }^{1,2}$, E. M. Hensor ${ }^{1,2}$, P. Emery ${ }^{1,2}$. 'Leeds Institute of Rheumatic and Musculoskeletal Medicine, University of Leeds; ${ }^{2}$ Leeds Biomedical Research Centre, National Institute of Health Research; ${ }^{3}$ Leeds Institute of Rheumatic and Musculoskeletal Medicine, Leeds University, Leeds, UK

Background: Selecting patients with new non-specific musculoskeletal complaints can enrich the prevalence of anti-cyclic citrullinated peptide (anti-CCP) antibodies compared with the general population. ${ }^{1}$ Patients with rheumatoid arthritis (RA) frequently present with involvement of the wrist, hands and feet. Patients with elderly onset RA have a higher frequency of polymyalgic onset. However, it is unknown if elderly patients with shoulder complaints are more likely to be CCP positive.

Objectives: To confirm the proportion of individuals with new-onset, non-specific MSK symptoms who were anti-CCP positive $(\mathrm{CCP}+)$ across a national cohort and investigate the initial presenting complaint of all individuals, as this may help determine whether there is a symptom complex that would prompt antibody testing. In addition to determine if the risk of being CCP + is increased in older patients presenting with shoulder symptoms.

Methods: Individuals aged $\geq 18$ years with new musculoskeletal complaints without synovitis from primary care were recruited prospectively. Participants completed a questionnaire on baseline musculoskeletal symptoms and provided a blood sample for anti-CCP antibody (Phadia CCP-2) testing. CCP+individuals where invited to attend follow-up in the rheumatology department, Leeds. The association between CCP status, smoking and shared epitope status was also assessed.

Results: 4257 individuals were recruited, 2.9\% (125/4257) were CCP+, a significantly higher proportion compared with the general population $(1 \%(95 \% \mathrm{Cl} 2.4 \%$ $>3.5 \% \mathrm{p}<0.001)$ ). Patients who presented with pain in the wrists, hands (RR 2.2 (1.5-2.9), $\mathrm{p}<0.001$ ) or feet (RR $1.72(1.2-2.4), \mathrm{p}<0.001)$ had an increased relative risk of being $\mathrm{CCP}+$. Patients who were older than 60 years who presented with shoulder symptoms $(4.8 \%(7 / 146))$ were no more likely to be CCP +than those who did not have shoulder symptoms $(3.2 \%(31 / 1007)$, chi square $p=0.313)$ and had the same prevalence of CCP +as those $<60$ years $(3.5 \%(13 / 370)$, chi square $\mathrm{p}=0.461)$. A significantly higher proportion of ever smokers were CCP+ $(14.2 \%$ (48/337)) compared with never smokers (3.3\% (64/1926); chi square $p<0.001)$. Ever smokers were also more likely than never smokers to be shared epitope positive in CCP +individuals $(62.2 \%(23 / 37)$ cf $37.8 \%(14 / 37) ; p<0.007)$.

Conclusions: Selecting individuals with new non-specific MSK symptoms without synovitis enriched the prevalence of anti-CCP positivity to $2.9 \%$. Patients presenting with symptoms localising to the wrists, hands and feet were more likely to be CCP + which could prompt anti-CCP testing in these patients in primary care. Patients with shoulder complaints were no more likely to be CCP +than those without shoulder symptoms and had the same prevalence of CCP +as those $<60$ years.

\section{REFERENCE:}

[1] Nam JL, et al., Enriching case selection for imminent RA: the use of antiCCP antibodies in individuals with new non-specific musculoskeletal symptoms - a cohort study. Ann Rheum Dis, 2016;75(8):1452-6.

Acknowledgements: The authors would like to thank all the participating general practitioners and health professionals and the UK Clinical Research Network teams for the referrals from primary care to the study
Disclosure of Interest: None declared

DOI: 10.1136/annrheumdis-2018-eular.5368

\section{FRI0047 1 MRI INTEROSSEOUS TENDON INFLAMMATION OCCURS IN ANTI-CCP POSITIVE AT-RISK INDIVIDUALS AND MAY PRECEDE THE DEVELOPMENT OF SYNOVITIS}

K. Mankia' ${ }^{1}$, M.A. D’Agostino ${ }^{1}$, E. Hensor ${ }^{2}$, E. Rowbotham ${ }^{3}$, L. Hunt ${ }^{1}$, I. Moller ${ }^{4}$, M. Miguel ${ }^{5}$, J.L. Nam ${ }^{1}$, A.L. Tan $^{1}$, J. Freeston ${ }^{6}$, L. Garcia-Montoya ${ }^{1}$, A. Grainger ${ }^{3}$, P. Emery'. ${ }^{1}$ Rheumatology, Leeds Institute of Rheumatic and Musculoskeletal Medicine and NIHR Leeds Biomedical Research Centre; ${ }^{2}$ Rheumatology, Leeds Institute of Rheumatic and Musculoskeletal Medicine; ${ }^{3}$ Radiology, Leeds Teaching Hospitals Trust and NIHR Leeds Biomedical Research Centre, Leeds, UK; ${ }^{4}$ Rheumatology; ${ }^{5}$ Anatomy, University of Barcelona, Barcelona, Spain;

${ }^{6}$ Rheumatology, Leeds Teaching Hospitals Trust and NIHR Leeds Biomedical Research Centre, Leeds, UK

Background: Tenosynovitis (TSV) occurs in individuals at-risk of developing RA and could explain pain and stiffness in the absence of synovitis. TSV of the wrist and finger flexor tendons has been described in at-risk individuals but involvement of other hand tendons has not been well investigated. The hand interossei are crucial to hand function and can become inflamed in RA $\left(.{ }^{1}\right.$ Whether the interosseous tendons (IT) are sites of inflammation in at-risk individuals, and how this relates to joint inflammation and clinical features is unknown.

Objectives: To describe the anatomy, prevalence, pattern and clinical associations of IT inflammation in anti-CCP positive at-risk individuals.

Methods: Anti-CCP positive individuals with no synovitis (CCP+), healthy controls $(\mathrm{HC})$, DMARD-naïve early RA patients (ERA) and treated 'late' RA patients (LRA) were recruited. All subjects underwent clinical and MRI assessment. 1.5T or 3T unilateral hand MRI scans were consensus scored for RAMRIS, TSV and IT inflammation by two radiologists. IT inflammation was defined as enhancing tissue around the tendon evident in two planes. For RAMRIS and tenosynovitis, scores were adjusted for 193 age-matched controls $\left({ }^{2}\right.$ To understand the anatomical basis for MRI IT inflammation, a cadaveric study was performed on 20 fresh hand specimens; coloured dyes were injected along the first dorsal IT and into the adjacent second MCP joint and specimens were frozen and sectioned.

Results: $93 \mathrm{CCP}+, 20 \mathrm{HC}, 47 \mathrm{ERA}$ and 28 LRA were recruited. Frequency of swollen and tender joints, MRI inflammation (synovitis, BME, erosions, TSV) and CRP level increased along the RA continuum with increasing disease duration. The proportion of patients with IT inflammation increased along the RA continuum No HC, 18/93 (19\%) CCP+, 23/47 (49\%) ERA and 16/28 (57\%) LRA patients had inflammation of $>1$ IT $(p<0.001)$. The number of affected ITs increased along the RA continuum $(p<0.001)$ and tendons associated with MCPJs 2 and 5 were most commonly affected. IT inflammation and MRI synovitis were associated with MCPJ swelling [OR $2.7(0.9,8.1)$ and OR $3.1(1.0,9.8)$ respectively] but IT inflammation was the only feature independently associated with MCPJ tenderness [OR $3.1(1.4,6.8) \mathrm{p}=0.004]$. In CCP+, 99/372 (27\%) MCPJs had only one MRI abnormality; in $68 \%$ of these the abnormality was extra-capsular (57\% TSV and $11 \%$ IT inflammation). No IT sheath was identified in the cadaveric specimens suggesting the MRI findings represent peri-tendonitis rather than TSV. Dye studies indicated no clear communication between the IT and the adjacent joint (figure 1).

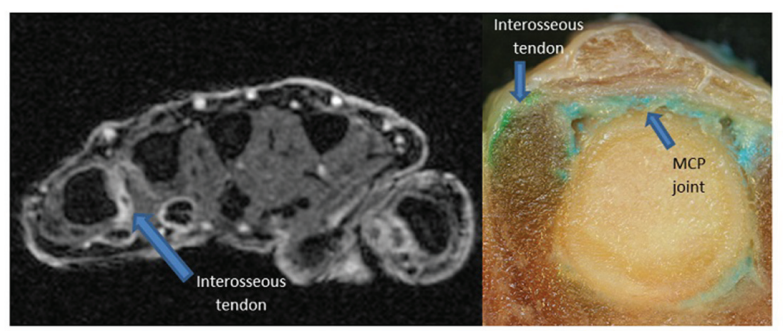

xial MRI showing inflammation of the $3^{\text {rd }}$ palmar interosseous tendon and frozen section showing green dye around the interosseous tendon and blue dye within the MCP joint

Abstract FRI0047 - Figure 1. Axial MRI showing inflammation of the 3rd palmar interosseous tendon and frozen section showing green dye around the interosseous tendon and blue dye within the MCP joint 
Conclusions: IT inflammation represents a peri-tendonitis and is present in anti$\mathrm{CCP}$ +at risk individuals and RA patients where it is associated with MCPJ swelling and tenderness. IT inflammation can occur as the lone MRI abnormality in $\mathrm{CCP}$ +at risk individuals suggesting the interossei may be an early extra-capsular target in the development of RA.

REFERENCES

[1] Rowbotham, et al. Eur Radiol 2015.

[2] Mangnus, et al. Arthritis Rheum 2016

Acknowledgements: D Glinatsi, M Ostergaard, P Bird

Disclosure of Interest: None declared

DOI: 10.1136/annrheumdis-2018-eular.5505

\section{\begin{tabular}{|l|l}
\hline FRI0048 MORTALITY AND MORBIDITY OF RHEUMATOID \\
\hline
\end{tabular} ARTHRITIS-ASSOCIATED LUNG DISEASE DURING A 10- YEAR PERIOD: A LONGITUDINAL COHORT STUDY OF 103 JAPANESE PATIENTS}

S. Shimoyama, Y. Fujieda, M. Kato, K. Oku, T. Bohgaki, O. Amengual, S. Yasuda, T. Atsumi. Department of Rheumatology, Endocrinology and Nephrology, Faculty of Medicine and Graduate School of Medicine, Hokkaido University, Sapporo-shi, Japan

Background: Subclinical and overt lung diseases associated with rheumatoid arthritis (RA-LD) are present in $30 \%-50 \%$ of the patients. Early and effective intervention improved joint prognosis in RA. By contrast, lung complications are still the primary contributors to premature deaths in patients with RA. Lung complication in RA can be due to a variety of conditions. However, the individual mortality and progression of pulmonary manifestations have not been established.

Objectives: To clarify the prognostic factors of patients with RA-LD.

Methods: This cohort study comprised RA patients examined with lung high resolution CT (HRCT) scan regardless of respiratory symptoms from 2005 to 2009 . Respiratory diagnoses were certified by pulmonologists. The patients were reas sessed by one follow-up CT scan after 10 years. All patients were evaluated for the events defined as death, serious infections and others (admission due to bone fracture, and ischaemic heart disease) from 2005 to 2017. Mortality risks were assessed using Kaplan-Meier method.

Results: Clinical features of 103 (82 females) patients are shown in table 1 Thirty-one (30\%) had RA-LD including 18 interstitial pneumonia and 13 bronchiolitis at the start of observation. Mean observation period was 110 months. During observation, patients without RA-LD (non RA-LD) never developed new lung complications. The 10 year survival rate (SR) was $92 \%$ (mortality rate was 1.3 per 100 patient-years) and the 10 year event free survival rate (EFS) $69 \%$ (event rate was 4.8 per 100 patient-years). SR in RA-LD was significantly low compared with non RA-LD ( $p=0.008$ ) (figure 1). EFS in RA-LD was significantly lower than in non RALD $(p=0.03)$. Types of lung complication didn't correlate with high mortality. The causes of death comprised infection (55\%), malignant tumour $(27 \%)$, interstitial pneumonia $(9 \%)$, and the others $(9 \%)$. The adverse events included infection (41\%), malignant tumour (21\%), bone fracture (15\%), cardiac disease $(10 \%)$, and severe drug eruption including Steven-Johnson syndrome (13\%). Univariate analysis showed that infection ( $p<0.001, H R 26.7)$ and acute exacerbation of RA-LD $(p<0.001$, HR 57.3, 95\% Cl 13-287) were strong risk factors for deaths.

Abstract FRI0048 - Table 1. Characteristics of RA patients with and without LD at the start of observation

\begin{tabular}{lcc}
\hline & RA-LD $(\mathrm{n}=31)$ & non RA-LD $(\mathrm{n}=72)$ \\
\hline Female $(\%)$ & 80 & 80 \\
age $(\mathrm{y})$ & $74 \pm 10$ & $68 \pm 13$ \\
age at RA diagnosis $(\mathrm{y})$ & $54 \pm 14$ & $48 \pm 13$ \\
RF positive $(\mathrm{n}, \%)$ & $20(65)$ & $48(67)$ \\
ACPA positive $(\mathrm{n}, \%)$ & $19(61)$ & $45(63)$ \\
DAS28 & $4.3 \pm 1.4$ & $4.4 \pm 1.5$ \\
Stage $(\mathrm{n}, \%)$ & $6(20)$ & $15(21)$ \\
Stage $(\mathrm{n}, \%)$ & $10(32)$ & $21(29)$ \\
Stage $(\mathrm{n}, \%)$ & $5(16)$ & $16(22)$ \\
Stage $(\mathrm{n}, \%)$ & $10(32)$ & $20(28)$ \\
\hline
\end{tabular}

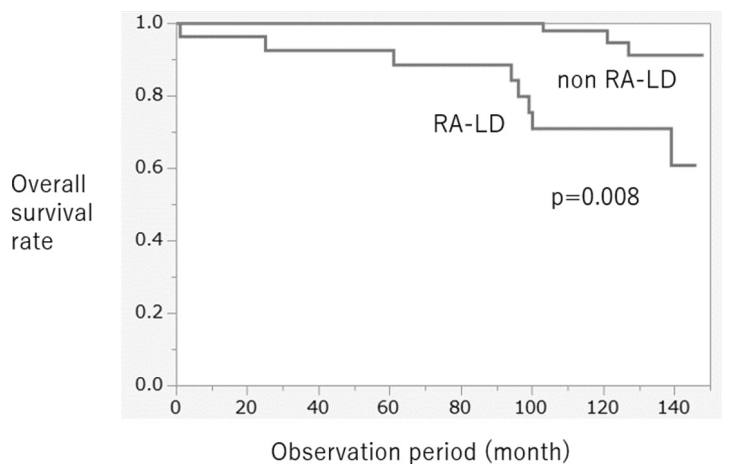

Conclusions: RA-LD is a serious complication in RA, and related with a high mortality.

Disclosure of Interest: None declared

DOI: 10.1136/annrheumdis-2018-eular.2884

\section{FRI0049 SIGNIFICANT IMPROVEMENT OF RHEUMATOID ARTHRITIS (RA) OUTCOME WITH REPEATED SELF- ASSESSMENT APPLYING SMART SYSTEM OF DISEASE MANAGEMENT (SSDM) MOBILES TOOLS: A COHORT STUDY OF RA PATIENTS EMPOWERING}

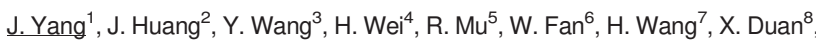

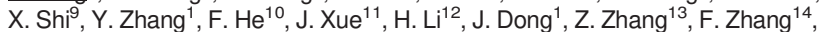
B. Wu ${ }^{15}$, H. Liu ${ }^{1}$, F. Xiao ${ }^{16}$, H. Xiao ${ }^{17}$, B. Wu ${ }^{17}$, Y. Liu' ${ }^{17}$, Y. Jia ${ }^{17}$, F. Zhang ${ }^{18}$. ${ }^{1}$ Department of rheumatology, Central Hospital of MianYang, Sichuan, Mianyang: ${ }^{2}$ Department of Rheumatology and Immunology, The sixth Affiliated Hospital of Sun Yat-sen University, Guangzhou; ${ }^{3}$ Department of Rheumatology and Immunology, The First Affiliated Hospital of BaoTou Medical College, Baotou; ${ }^{4}$ Department of rheumatology, Northern Jiangsu People's Hospital, Yangzhou; ${ }^{5}$ Department of Rheumatology and Immunology, People's Hospital, Beijing University Medical School, Beijing; ${ }^{6}$ Department of rheumatology, Central Hospital of XinXiang, Xingxiang; ${ }^{7}$ Department of Rheumatology and Immunology, JiaXing First Hospital, Zhejiang, Jiaxing; ${ }^{8}$ Department of rheumatology, The Second Affiliated Hospital of Nanchang University, Nanchang; ${ }^{9}$ Department of Rheumatology and Immunology, The First Affiliated Hospital of Henan University of Science and Technology, Luoyang; ${ }^{10}$ Department of rheumatology, Suining Central Hospital, Suining; ${ }^{11}$ Department of Rheumatology and Immunology, The Second Affiliated Hospital of Zhejiang University School of Medicine, Hangzhou; ${ }^{12}$ Department of rheumatology, The Affiliated Hospital of Inner Mongolia Medical University, Hohhot, ${ }^{13}$ Department of Rheumatology and Immunology, Linyi people's Hospital, Linyi; ${ }^{14}$ Department of Rheumatology and Immunology, Hebei General Hospital, Shijiazhuang; ${ }^{15}$ Department of rheumatology, Chongqing Hospital of Traditional Chinese Medicine, Chongqing; ${ }^{16}$ Suzhou antiinflammatory research institue, Suzhou; ${ }^{17}$ Shanghai Gothic Internet Technology Co., Ltd., Shanghai; ${ }^{18}$ Department of Rheumatology and Immunology, Peking Union Medical College hospital, Beijing, China

Background: Treat-to-Target (T2T) strategy are critical for the treatment of RA but the Chinese rheumatologists can hardly provide patients with a complete assessment in the clinic due to limited time. The SSDM includes interfaces of both physicians' and patients' application. After inputting lab test records, treatment regiments, and executing DAS28 assessment by patients themselves, all data can be synchronised automatically to the authorised physicians' mobile tool. The rheumatologists can adjust treatment regiments base on patients' profile. Our previous study showed that patients in China can master the application of SSDM for accurately evaluating DAS28 and health assessment questionnaire (HAQ) after training.

Objectives: The objective of this study is to explore the effectiveness of applying SSDM in improvement of disease activity after repeated self-assessment in Chinese RA patients.

Methods: Patients were trained to do DAS28 evaluation with SSDM and asked to repeat the self-assessment once a month. Descriptive statistics were performed 